\title{
A Comparative Study of the Results of Three cm versus Six Cm Distance from Pyloric Sphincter in Sleeve Gastrectomy
}

\author{
Ahmed Maher *, Magdy Mahmoud Mostafa, Mohammad Arafat Abd El-Maksoud \\ Department of General Surgery, Faculty of Medicine, Al-Azhar University, Cairo, Egypt \\ * Corresponding author: Ahmed Maher, Bab El-Shaaria Hospital, E-mail: dactaraaaa@ hotmail.com
}

\begin{abstract}
Background: Obesity is excessive fat accumulation. Now, laparoscopic sleeve gastrectomy (LSG) is gaining popularity as a primary, staged and provisional operation for its proven safety and simplicity. Complete removal of the gastric fundus makes adequate weight loss and plays a key role in reducing co-morbidities, ghrelin production, and eliminate inlet of the stomach to become small enough so that the patient will feel full quickly.

Objective: The aim of this study was to assess the results of resecting the stomach $6-\mathrm{cm}$ versus $3-\mathrm{cm}$ from the pylorus regarding weight loss, reflux development, nausea and vomiting

Patients and Methods: This randomly selected prospective study included a total of 30 morbidly obese patients, attending at Department of General Surgery, Bab El-Shaaria, and Al-Azhar University Hospital for doing sleeve gastrectomy. According to the starting point of stomach resection, the included subjects were randomly divided into two groups; each was 15 patients. Group (A) started 3 $\mathrm{cm}$ from the pylorus towards the gastro-esophageal junction and Group (B) started $6 \mathrm{~cm}$ from the pylorus. These patients followed up over a period of 2 years for postoperative nausea; vomiting and reflux symptoms and their weight loss.

Results: group A patients ( $3 \mathrm{~cm}$ groups) were $>14$ times at a higher risk to have minor complications in the form of nausea; vomiting and reflux compared to group B patients $(6 \mathrm{~cm}$ groups) without any difference between both groups regarding BMI changes over a period of six months.

Conclusion: It could be concluded that leaving an antral pouch of $6 \mathrm{~cm}$ size while performing this procedure is recommended for better outcome with minor complications.
\end{abstract}

Keywords: Obesity, laparoscopic sleeve gastrectomy, bariatric surgery

\section{INTRODUCTION}

Obesity is excessive fat accumulation, and not simply being overweight. The average human body usually consists of $18 \%$ body fat, which in essence is energy stored for fasting and starvation times. Thus, obesity can be defined as 'over-storage of body fat beyond 18\%'. Usually, body fat above $30 \%$ is considered obesity. According to this definition, obesity should be judged by measuring stored fat in the body ${ }^{[1]}$.

At present, obesity can be evaluated by three methods: (1) Comparison with standard body weight, (2) Physique index and (3) Measurement of subcutaneous fat thickness ${ }^{[17]}$ .Sleeve gastrectomy (SG) was introduced as a multi-purpose bariatric operation ${ }^{[2]}$. SG involves removing the fundus and greater curvature portion of the stomach, leaving only a lesser curvature tube ${ }^{[3]}$.

SG was initially described as a first-step procedure followed by either bilio-pancreatic diversion with duodenal switch (BPD-DS) or
Roux-en-Y gastric bypass (RYGB) in supersuper obese patients, body mass index (BMI) $>60 \mathrm{Kg} / \mathrm{m} 2$ or in high-risk patients ${ }^{[4,5]}$. SG has been indicated as a definitive treatment in patients with BMI $>40 \mathrm{~kg} / \mathrm{m}^{2}$ or BMI $>35$ $\mathrm{kg} / \mathrm{m}^{2}$ associated with co-morbidities ${ }^{[6]}$ It has also been proposed for patients with moderate obesity BMI $<35 \mathrm{~kg} / \mathrm{m}^{2}$ and metabolic syndrome ${ }^{[7]}$.

The aim of this study was to find out the results of resecting the stomach $6-\mathrm{cm}$ versus $3-\mathrm{cm}$ from the pylorus regarding weight loss, reflux development, nausea and vomiting.

\section{PATIENTS AND METHODS}

This randomly selected prospective study included a total of 30 morbidly obese patients, aged 17 to 60 years, attending at Department of General Surgery, Bab El-Shaaria, Al-Azhar University Hospital for doing sleeve gastrectomy. Written informed consent from all 
the subjects were obtained. This study was conducted between January 2016 and December 2017.

Ethical approval:

The study was approved by the Ethics Board of Al-Azhar University and an informed written consent was taken from each participant in the study.

According to the starting point of stomach resection, the included subjects were randomly divided into two groups; each was 15 patients. Group (A) started $3 \mathrm{~cm}$ from the pylorus towards the gastro-esophageal junction and Group (B) started $6 \mathrm{~cm}$ from the pylorus. These patients followed up over a period of 2 years for postoperative nausea; vomiting and reflux symptoms and their weight loss.

\section{Inclusion criteria:}

1. Patients who have BMI $40 \mathrm{~kg} / \mathrm{m}^{2}$ or more, or between 35 and $40 \mathrm{~kg} / \mathrm{m}^{2}$ with other significant disease that could be improved if they lost weight.

2. Both sexes (males and females)

3. Patients generally fit for anesthesia and surgery.

4. Patients committed to the need for follow up.

\section{Exclusion criteria:}

1. Patients with previous abdominal surgeries.

2. Patients with psychiatric problems.

3. Severe cardiopulmonary disease or other serious organic disease making the subject a high-risk surgical candidate, uncontrolled hypertension, and portal hypertension.

4. Pregnancy or lactation at surgery.

5. Drug or alcohol abuse.

6. Previous malabsorptive or restrictive procedures performed for the treatment of obesity.

During the surgery, Marking of the distance from the pyloric ring to the starting point of resection was done; where a calibrated string was used to determine the starting point of resection from the pylorus either $3 \mathrm{~cm}$ or $6 \mathrm{~cm}$. All patients were examined monthly during the first six months for BMI changes and postoperative complications mainly (nausea, vomiting and reflux) where nausea and vomiting were categorized by a scoring system called PONV (post-operative nausea and vomiting scoring system)

Statistical analysis: The data was coded and entered using the statistical package SPSS version 15. The data was summarized using number and percentage for qualitative values. Statistical differences between groups were tested using Chi Square test for qualitative variables. Logistic regression analysis was done to test for significant predictors of postoperative complications. P- Values less than or equal to 0.05 were considered statistically significant.

\section{RESULTS}

This was a randomly selected prospective study carried out on morbidly obese patients where thirty patients underwent sleeve gastrectomy. These patients grouped into two groups (each group is 15 patients) according to the starting point of resection of the stomach; group (A) started $3 \mathrm{~cm}$ from the pylorus towards the gastro-esophageal junction and group (B) $6 \mathrm{~cm}$ from the pylorus.

\section{(A) Age and sex distribution}

Age:

The ages of patient ranged from 17 to 60 years old with mean of 34.5 years. The majority of patients in this study were in the age group $21-40(63.3 \%)$ with five patients $(6.7 \%)$ below twenty years, as shown in Table (1).

Table (1): Age distribution

\begin{tabular}{|c|c|c|c|}
\hline & $\begin{array}{l}\text { Group A } \\
3 \mathrm{~cm}\end{array}$ & $\begin{array}{l}\text { Group B } \\
6 \mathrm{~cm}\end{array}$ & Total \\
\hline \multicolumn{4}{|l|}{$\begin{array}{l}\text { Age } \\
\text { (years) }\end{array}$} \\
\hline$<20$ & $\begin{array}{l}0 \\
(0 \%)\end{array}$ & $\begin{array}{l}2 \\
(13.4 \%)\end{array}$ & $\begin{array}{l}2 \\
(6.7 \%)\end{array}$ \\
\hline $21-40$ & $\begin{array}{l}9 \\
(60 \%)\end{array}$ & $\begin{array}{l}10 \\
(66.6 \%)\end{array}$ & $\begin{array}{l}19 \\
(63.3 \%)\end{array}$ \\
\hline $41-60$ & $\begin{array}{l}4 \\
(26.6 \%)\end{array}$ & $\begin{array}{l}3 \\
(20 \%)\end{array}$ & $\begin{array}{l}7 \\
(23.3 \%)\end{array}$ \\
\hline$>60$ & $\begin{array}{l}2 \\
(13.4 \%)\end{array}$ & $\begin{array}{l}0 \\
(.0 \%)\end{array}$ & $\begin{array}{l}2 \\
(6.7 \%)\end{array}$ \\
\hline Total & $\begin{array}{l}15 \\
100.0 \%\end{array}$ & $\begin{array}{l}15 \\
100.0 \%\end{array}$ & $\begin{array}{l}30 \\
100.0 \%\end{array}$ \\
\hline
\end{tabular}

SEX: 
The majority of candidates in this study $(73.4 \%)$ were females, as shown in Table (5).

Table (2): Sex distribution of patients

\begin{tabular}{|c|c|c|c|}
\hline & $\begin{array}{l}\text { Group A } \\
\\
\text { cm }\end{array}$ & $\begin{array}{r}\text { Group B } \\
6 \mathrm{~cm}\end{array}$ & Total \\
\hline Sex & & & \\
\hline Male & $\begin{array}{l}4 \\
(26.7 \%)\end{array}$ & $\begin{array}{l}4 \\
(26.7 .0 \%)\end{array}$ & $\begin{array}{l}8 \\
(26.7 \%)\end{array}$ \\
\hline$\overline{\text { Female }}$ & $\begin{array}{l}11 \\
(73.3 \%)\end{array}$ & $\begin{array}{l}11 \\
(73.3 \%)\end{array}$ & $\begin{array}{l}22 \\
(73.3 \%)\end{array}$ \\
\hline Total & $\begin{array}{l}15 \\
(100.0 \%)\end{array}$ & $\begin{array}{l}15 \\
(100.0 \%)\end{array}$ & $\begin{array}{l}30 \\
(100.0 \%)\end{array}$ \\
\hline
\end{tabular}

Marital status:

Most of the candidates in our study were married $(60 \%)$ as compared to single (40\%), as shown in Table (3).

Table (3): Marital Status of patients

\begin{tabular}{|c|c|c|c|}
\hline & $\begin{array}{l}\text { Group A } \\
3 \mathrm{~cm}\end{array}$ & $\begin{array}{l}\text { Group B } \\
6 \mathrm{~cm}\end{array}$ & Total \\
\hline \multicolumn{4}{|l|}{$\begin{array}{l}\text { Marital } \\
\text { Status }\end{array}$} \\
\hline Married & $\begin{array}{l}8 \\
(53.4 \%)\end{array}$ & $\begin{array}{l}10 \\
(66.6 \%)\end{array}$ & $\begin{array}{l}18 \\
(60.0 \%)\end{array}$ \\
\hline Single & $\begin{array}{l}7 \\
(46.6 \%)\end{array}$ & $\begin{array}{l}5 \\
(33.4 \%)\end{array}$ & $\begin{array}{l}12 \\
(40.0 \%)\end{array}$ \\
\hline Total & $\begin{array}{l}15 \\
(100.0 \%)\end{array}$ & $\begin{array}{l}15 \\
(100.0 \%)\end{array}$ & $\begin{array}{l}30 \\
(100.0 \%)\end{array}$ \\
\hline
\end{tabular}

\section{Occupational Status}

The majority of subjects in our study $(53.4 \%)$ were working, as shown in Table (4).

Table (4): Occupational Status of patients

\begin{tabular}{|c|c|c|c|}
\hline & $\begin{array}{l}\text { Group } \\
\text { A } \\
3 \mathrm{~cm}\end{array}$ & $\begin{array}{l}\text { Group } \\
\text { B } \\
6 \mathrm{~cm} \\
\end{array}$ & Total \\
\hline $\begin{array}{l}\text { Occupationa } \\
\text { I Status }\end{array}$ & & & \\
\hline Working & $\begin{array}{l}8 \\
(53.4 \%)\end{array}$ & $\begin{array}{l}8 \\
(53.4 \%)\end{array}$ & $\begin{array}{l}16 \\
(53.4 \%)\end{array}$ \\
\hline Not working & $\begin{array}{l}7 \\
(46.6 \%)\end{array}$ & $\begin{array}{l}7 \\
(46.6 \%)\end{array}$ & $\begin{array}{l}14 \\
(46.6 \%)\end{array}$ \\
\hline Total & $\begin{array}{l}15 \\
(100.0 \% \\
)\end{array}$ & $\begin{array}{l}15 \\
(100.0 \%\end{array}$ & $\begin{array}{l}30 \\
(100.0 \% \\
)\end{array}$ \\
\hline
\end{tabular}

(B) Patients' initial BMIs and Feeding habits Fifty percent of patients in this study had BMI $>50,26.7 \%$ had BMI $40-45 \%$ and $3.3 \%$ had
BMI 35-40\% with co-morbidities, as shown in Table (5).

Table (5): Initial BMI of patients

\begin{tabular}{|c|c|c|c|}
\hline & $\begin{array}{l}\text { Group A } \\
3 \mathrm{~cm}\end{array}$ & $\begin{array}{l}\text { Group B } \\
6 \mathrm{~cm}\end{array}$ & Total \\
\hline $\begin{array}{l}\text { Initial } \\
\text { BMI } \\
\left(\mathrm{kg} / \mathrm{m}^{2}\right)\end{array}$ & & & \\
\hline $35-40$ & $\begin{array}{l}1 \\
(6.6 \%)\end{array}$ & $\begin{array}{l}0 \\
(0 \%)\end{array}$ & $\begin{array}{l}1 \\
(3.3 \%)\end{array}$ \\
\hline $40-45$ & $\begin{array}{l}4 \\
(26.7 \%)\end{array}$ & $\begin{array}{l}4 \\
(26.7 \%)\end{array}$ & $\begin{array}{l}8 \\
(26.7 \%)\end{array}$ \\
\hline $45-50$ & $\begin{array}{l}4 \\
(26.7 \%)\end{array}$ & $\begin{array}{l}2 \\
(13.3 \%)\end{array}$ & $\begin{array}{l}6 \\
(20.0 \%)\end{array}$ \\
\hline$>50$ & $\begin{array}{l}6 \\
(40 \%)\end{array}$ & $\begin{array}{l}9 \\
(60 \%)\end{array}$ & $\begin{array}{l}15 \\
(50.0 \%)\end{array}$ \\
\hline Total & $\begin{array}{l}15 \\
(100.0 \%)\end{array}$ & $\begin{array}{l}15 \\
(100.0 \%)\end{array}$ & $\begin{array}{l}30 \\
(100.0 \%)\end{array}$ \\
\hline
\end{tabular}

The majority of patients in this study were bulky eater $(76.7 \%)$ versus $(23.3 \%)$ were sweet eater, as shown in Table (6).

Table (6): Feeding habits of patients

\begin{tabular}{|l|l|l|l|}
\hline \multicolumn{4}{|c|}{$\begin{array}{l}\text { Group A } \\
\mathbf{3} \text { cm }\end{array}$} \\
\hline $\begin{array}{l}\text { Feeding } \\
\text { habits }\end{array}$ & $\begin{array}{l}\text { Group B } \\
\mathbf{c m}\end{array}$ & Total \\
\hline $\begin{array}{l}\text { Bulky } \\
\text { eater }\end{array}$ & $\begin{array}{l}12 \\
(80 \%)\end{array}$ & $\begin{array}{l}11 \\
(73.3 \%)\end{array}$ & $\begin{array}{l}23 \\
(76.7 \%)\end{array}$ \\
\hline $\begin{array}{l}\text { Sweet } \\
\text { eater }\end{array}$ & 3 & 4 & 7 \\
\hline Total & $\begin{array}{l}15 \\
(100.0 \%)\end{array}$ & $\begin{array}{l}15 \\
(100.0 \%)\end{array}$ & $\begin{array}{l}30 \\
(100.0 \%)\end{array}$ \\
\hline
\end{tabular}

\section{(C) Associated obesity related diseases}

D.M:

Ninety percent of the patients in this study were not diabetic versus $10 \%$ were diabetic on oral hypoglycemic drugs, as shown in Table (7).

Table (7): Presence of Diabetes Mellitus in our patients

\begin{tabular}{|c|c|c|c|}
\hline & $\begin{array}{l}\text { Group A } \\
3 \mathrm{~cm}\end{array}$ & $\begin{array}{l}\text { Group B } \\
6 \mathrm{~cm}\end{array}$ & Total \\
\hline \multicolumn{4}{|l|}{ Diabetes } \\
\hline Diabetic & $\begin{array}{l}1 \\
(6.6 \%)\end{array}$ & $\begin{array}{l}2 \\
(13.4 \%)\end{array}$ & $\begin{array}{l}3 \\
(10.0 \%)\end{array}$ \\
\hline Not & $\begin{array}{l}14 \\
(93.4 \%)\end{array}$ & $\begin{array}{l}13 \\
(86.6 \%)\end{array}$ & $\begin{array}{l}27 \\
(90.0 \%)\end{array}$ \\
\hline Total & $\begin{array}{l}15 \\
(100.0 \%)\end{array}$ & $\begin{array}{l}15 \\
(100.0 \%)\end{array}$ & $\begin{array}{l}30 \\
(100.0 \%)\end{array}$ \\
\hline
\end{tabular}

\section{Hypertension:}


Eighty percent of subjects in our study were not hypertensive versus twenty percent were hypertensive and on medication, as shown in Table (8).

Table (8): Presence of Hypertension in our patients

\begin{tabular}{|l|l|l|l|}
\hline \multicolumn{1}{|c}{} & \multicolumn{1}{l}{$\begin{array}{l}\text { Group } \\
\text { A cm }\end{array}$} & \multicolumn{2}{l|}{$\begin{array}{l}\text { Group } \\
\text { B cm }\end{array}$} \\
\hline $\begin{array}{l}\text { Hypertensio } \\
\text { n cm }\end{array}$ & & & \\
\hline $\begin{array}{l}\text { Hypertensiv } \\
\text { e }\end{array}$ & 3 & 2 & 5 \\
\hline Not & $\begin{array}{l}12 \\
(80 \%)\end{array}$ & $\begin{array}{l}13 \\
(86.6 \%)\end{array}$ & $\begin{array}{l}25 \\
(83.3 \%)\end{array}$ \\
\hline Total & $\begin{array}{l}15 \\
(100.0 \%\end{array}$ & $\begin{array}{l}15 \\
(100.0 \%\end{array}$ & $\begin{array}{l}30 \\
(100.0 \%\end{array}$ \\
& ) & ) & ) \\
\hline
\end{tabular}

\section{Other chronic diseases:}

83.3 percent of patients in this study were not complaining from chronic diseases (in the form of chest diseases, liver, cardiac, renal or other medical disorders) versus $16.7 \%$ suffered from chronic diseases mostly chest diseases and two patients had poliomyelitis, as shown in Table (9).

Table (9): Presence of chronic diseases in our patients

\begin{tabular}{|l|l|l|l|}
\hline \multicolumn{1}{|c}{$\begin{array}{l}\text { Group A } \\
\mathbf{3 ~ c m}\end{array}$} & \multicolumn{1}{l}{$\begin{array}{l}\text { Group B } \\
\mathbf{6 ~ c m}\end{array}$} & Total \\
\hline $\begin{array}{l}\text { Chronic } \\
\text { disease }\end{array}$ & & & \\
\hline Present & 3 & $\begin{array}{l}2 \\
(13.4 \%)\end{array}$ & $\begin{array}{l}5 \\
(16.7 \%)\end{array}$ \\
\hline Not & $\begin{array}{l}(20 \%) \\
(80 \%)\end{array}$ & $\begin{array}{l}13 \\
(86.6 \%)\end{array}$ & $\begin{array}{l}25 \\
(83.3 \%)\end{array}$ \\
\hline Total & $\begin{array}{l}15 \\
(100.0 \%)\end{array}$ & $\begin{array}{l}15 \\
(100.0 \%)\end{array}$ & $\begin{array}{l}30 \\
(100.0 \%)\end{array}$ \\
\hline
\end{tabular}

\section{Weight loss and patient satisfaction:}

The overall patients' weight loss percentage ranged from 30 to $86.9 \%$ excess body weight loss with a mean of $60 \%$. In group A, patients' weight loss percentage ranged from 31.2 to $86.9 \%$ excess body weight loss with a mean of $60.9 \%$, however, in group B, patients' weight loss percentage ranged from 30 to $83.5 \%$ excess body weight loss with a mean of $61.1 \%$, as shown in Table (13).
Table (10): Range and mean of weight loss at 6 months

\begin{tabular}{|c|c|c|c|}
\hline & $\begin{array}{l}\text { Group } \\
\text { A } \\
3 \mathrm{~cm}\end{array}$ & $\begin{array}{l}\text { Group } \\
\text { B } \\
6 \mathrm{~cm}\end{array}$ & $\begin{array}{l}\text { All } \\
\text { Patients }\end{array}$ \\
\hline \multicolumn{4}{|l|}{ Weight loss } \\
\hline Minimum & $31.2 \%$ & $30 \%$ & $30 \%$ \\
\hline Maximum & $86.9 \%$ & $83.5 \%$ & $86.9 \%$ \\
\hline Mean & $60.9 \%$ & $61.1 \%$ & $60 \%$ \\
\hline
\end{tabular}

In this study, (40\%) of patients lost (40-60\%) of their excess body weight within 6 months without significance to any group. However, in this study, two patients (13.4\%) in group A lost more than $80 \%$ of their excess body weight over a period of six months versus one patient in group B lost $80 \%$ of his excess body weight, as shown in Table (14).

Table (11): Percentage of weight loss at 6 months

\begin{tabular}{|c|c|c|c|}
\hline & $\begin{array}{l}\text { Group A } \\
3 \mathrm{~cm}\end{array}$ & $\begin{array}{l}\text { Group B } \\
6 \mathrm{~cm}\end{array}$ & Total \\
\hline \multicolumn{4}{|c|}{$\begin{array}{l}\text { Percentage } \\
\text { of weight } \\
\text { loss at } 6 \\
\text { months }(\%)\end{array}$} \\
\hline $20-40$ & $\begin{array}{l}1 \\
(6.6 \%)\end{array}$ & $\begin{array}{l}1 \\
(6.6 \%)\end{array}$ & $\begin{array}{l}2 \\
(6.7 \%) \\
\end{array}$ \\
\hline $41-60$ & $\begin{array}{l}6 \\
(40 \%)\end{array}$ & $\begin{array}{l}6 \\
(40 \%)\end{array}$ & $\begin{array}{l}12 \\
(40 \%)\end{array}$ \\
\hline $61-80$ & $\begin{array}{l}6 \\
(40 \%)\end{array}$ & $\begin{array}{l}7 \\
(46.8 \%)\end{array}$ & $\begin{array}{l}13 \\
(43.3 \%)\end{array}$ \\
\hline$>80$ & $\begin{array}{l}2 \\
(13.4 \%)\end{array}$ & $\begin{array}{l}1 \\
(6.6 \%)\end{array}$ & $\begin{array}{l}3 \\
(10 \%)\end{array}$ \\
\hline Total & $\begin{array}{l}15 \\
(100.0 \%)\end{array}$ & $\begin{array}{l}15 \\
(100.0 \%)\end{array}$ & $\begin{array}{l}30 \\
(100.0 \%)\end{array}$ \\
\hline
\end{tabular}

The majority of our patients (90\%) satisfied from the procedure and its results without any privileges for any group $(3 \mathrm{~cm}$ or $6 \mathrm{~cm})$ and with the presence of minor complications, which had accepted, by most of them, as shown in Table (15).

Table (12): Patients satisfaction

\begin{tabular}{|c|c|c|c|}
\hline & $\begin{array}{l}\text { Group A } \\
3 \mathrm{~cm}\end{array}$ & $\begin{array}{l}\text { Group B } \\
6 \mathrm{~cm}\end{array}$ & Total \\
\hline \multicolumn{4}{|c|}{ Satisfaction } \\
\hline Satisfied & $\begin{array}{l}13 \\
(86.6 \%)\end{array}$ & $\begin{array}{l}14 \\
(93.4 \%)\end{array}$ & $\begin{array}{l}27 \\
(90 \%)\end{array}$ \\
\hline $\begin{array}{l}\text { Partially } \\
\text { satisfied }\end{array}$ & $\begin{array}{l}2 \\
(13.4 \%)\end{array}$ & $\begin{array}{l}1 \\
(6.6 \%)\end{array}$ & $\begin{array}{l}3 \\
(10 \%)\end{array}$ \\
\hline $\begin{array}{l}\text { Not } \\
\text { satisfied }\end{array}$ & $\begin{array}{l}0 \\
(0 \%)\end{array}$ & $\begin{array}{l}0 \\
(0 \%)\end{array}$ & $\begin{array}{l}0 \\
(0 \%)\end{array}$ \\
\hline Total & $\begin{array}{l}15 \\
(100.0 \%)\end{array}$ & $\begin{array}{l}15 \\
(100.0 \%)\end{array}$ & $\begin{array}{l}30 \\
(100.0 \%)\end{array}$ \\
\hline
\end{tabular}




\section{Appetite postoperative:}

Almost all of patients underwent this operation with either techniques $(3 \mathrm{~cm}$ or $6 \mathrm{~cm})$ showed marked reduction in their appetite $(96.7 \%)$, as shown in Table (13).

Table (13): Appetite postoperative

\begin{tabular}{|c|c|c|c|}
\hline & $\begin{array}{l}\text { Group A } \\
3 \mathrm{~cm}\end{array}$ & $\begin{array}{l}\text { Group B } \\
6 \mathrm{~cm}\end{array}$ & Total \\
\hline \multicolumn{4}{|l|}{ Appetite } \\
\hline Decreased & $\begin{array}{l}15 \\
(100 \%)\end{array}$ & $\begin{array}{l}14 \\
(93.4 \%)\end{array}$ & $\begin{array}{l}29 \\
(96.7 \%)\end{array}$ \\
\hline Stationary & $\begin{array}{l}0 \\
(0 \%)\end{array}$ & $\begin{array}{l}1 \\
(6.6 \%)\end{array}$ & $\begin{array}{l}1 \\
(3.3 \%)\end{array}$ \\
\hline Total & $\begin{array}{l}15 \\
(100.0 \%)\end{array}$ & $\begin{array}{l}15 \\
(100.0 \%)\end{array}$ & $\begin{array}{l}30 \\
(100.0 \%)\end{array}$ \\
\hline
\end{tabular}

\section{(F) Complications}

1) General considerations related to complications:

In this study, there was no major complications (e.g.; leakage, bleeding, pulmonary embolism or death). However, minor complications in the form of nausea, vomiting and reflux were more with $3 \mathrm{~cm}$ group (100\%) as compared to $6 \mathrm{~cm}$ group (66.6\%). as shown in Table (14)

Table (14): Correlation between two groups and presence of Minor complications (nausea, vomiting and reflux)

\begin{tabular}{|c|c|c|c|}
\hline & $\begin{array}{l}\text { Group } \\
\text { A } \\
3 \text { cm } \\
\end{array}$ & $\begin{array}{l}\text { Group } \\
\text { B } \\
6 \mathrm{~cm} \\
\end{array}$ & Total \\
\hline $\begin{array}{l}\text { Minor } \\
\text { complication } \\
\text { s }\end{array}$ & & & \\
\hline No & $\begin{array}{l}0 \\
(0 \%)\end{array}$ & $\begin{array}{l}5 \\
(33.4 \%)\end{array}$ & $\begin{array}{l}5 \\
(16.7 \%)\end{array}$ \\
\hline yes & $\begin{array}{l}15 \\
(100 \%)\end{array}$ & $\begin{array}{l}10 \\
(66.6 \%)\end{array}$ & $\begin{array}{l}25 \\
(83.3 \%)\end{array}$ \\
\hline Total & $\begin{array}{l}15 \\
(100.0 \%\end{array}$ & $\begin{array}{l}15 \\
(100.0 \%\end{array}$ & $\begin{array}{l}30 \\
(100.0 \%\end{array}$ \\
\hline
\end{tabular}

\section{2) Postoperative Reflux:}

There was no statistical difference between both groups $(6 \mathrm{~cm}$ and $3 \mathrm{~cm})$ regarding postoperative reflux, where most of patients $(60 \%)$ didn't suffer reflux symptom versus $(40 \%)$ who suffered from reflux. In addition, (46.6\%) of patients in $3 \mathrm{~cm}$ group suffered from reflux versus $(33.4 \%)$ in $6 \mathrm{~cm}$ group who suffered from reflux taking in consideration that the sample size is thirty patients so higher sample size may confirm this correlation, as shown in Table (15)

Table (16): Correlation between two groups and presence of Postoperative Reflux

\begin{tabular}{|c|c|c|c|}
\hline & $\begin{array}{l}\text { Group A } \\
3 \mathrm{~cm}\end{array}$ & $\begin{array}{l}\text { Group B } \\
6 \mathrm{~cm}\end{array}$ & Total \\
\hline \multicolumn{4}{|l|}{ Reflux } \\
\hline Absent & $\begin{array}{l}8 \\
(53.4 \%)\end{array}$ & $\begin{array}{l}10 \\
(66.6 \%)\end{array}$ & $\begin{array}{l}18 \\
(60 \%)\end{array}$ \\
\hline Presence & $\begin{array}{l}7 \\
(46.6 \%) \\
\end{array}$ & $\begin{array}{l}5 \\
(33.4 \%) \\
\end{array}$ & $\begin{array}{l}12 \\
(40 \%) \\
\end{array}$ \\
\hline Total & $\begin{array}{l}15 \\
(100.0 \%)\end{array}$ & $\begin{array}{l}15 \\
(100.0 \%)\end{array}$ & $\begin{array}{l}30 \\
(100.0 \%)\end{array}$ \\
\hline
\end{tabular}

3) Vomiting score: as shown in Table (17)

Table (17): Correlation between two groups and presence of vomiting

\begin{tabular}{|c|c|c|c|}
\hline & $\begin{array}{l}\text { Group A } \\
3 \mathrm{~cm}\end{array}$ & $\begin{array}{l}\text { Group B } \\
6 \mathrm{~cm}\end{array}$ & Total \\
\hline \multicolumn{4}{|l|}{$\begin{array}{l}\text { Vomiting } \\
\text { score } \\
\text { (daily) } \\
\end{array}$} \\
\hline No & $\begin{array}{l}3 \\
(20 \%)\end{array}$ & $\begin{array}{l}7 \\
(46.6 \%)\end{array}$ & $\begin{array}{l}10 \\
(33.3 \%)\end{array}$ \\
\hline Once & $\begin{array}{l}5 \\
(33.3 \%)\end{array}$ & $\begin{array}{l}3 \\
(20 \%)\end{array}$ & $\begin{array}{l}8 \\
(26.6 \%)\end{array}$ \\
\hline Twice & $\begin{array}{l}5 \\
(33.3 \%)\end{array}$ & $\begin{array}{l}4 \\
(26.6 \%)\end{array}$ & $\begin{array}{l}9 \\
(30 \%)\end{array}$ \\
\hline $\begin{array}{l}\text { Three } \\
\text { times and } \\
\text { more }\end{array}$ & $\begin{array}{l}2 \\
(13.3 \%)\end{array}$ & $\begin{array}{l}1 \\
(6.6 \%)\end{array}$ & $\begin{array}{l}3 \\
(10.0 \%)\end{array}$ \\
\hline Total & $\begin{array}{l}15 \\
(100.0 \%)\end{array}$ & $\begin{array}{l}15 \\
(100.0 \%)\end{array}$ & $\begin{array}{l}30 \\
(100.0 \%)\end{array}$ \\
\hline
\end{tabular}

\section{Predictors:}

1) Predictors for vomiting:

$3 \mathrm{~cm}$ groups were $>6$ times at a higher risk to have vomiting > once compared to $6 \mathrm{~cm}$ group, as shown in Tables (18).

Table (18): Predictors for vomiting (> once)

\begin{tabular}{|c|c|c|c|c|}
\hline & $\begin{array}{l}\text { p- } \\
\text { value } \\
\text { Lower }\end{array}$ & $\begin{array}{l}\text { Odds } \\
\text { ratio } \\
\text { Upper }\end{array}$ & $\begin{array}{l}95.0 \% \\
\text { odds ra } \\
\text { Lower }\end{array}$ & $\begin{array}{l}\text { C.I. for } \\
\text { io } \\
\text { Upper }\end{array}$ \\
\hline $\begin{array}{l}\text { Groups } \\
(3 \mathrm{~cm} / 6 \\
\mathrm{cm})\end{array}$ & 0.011 & 6.255 & 1.527 & 25.620 \\
\hline Constant & 0.261 & 1.545 & & \\
\hline
\end{tabular}

2) Predictors for minor complications: 
$3 \mathrm{~cm}$ groups were $>14$ times at a higher risk to have minor complications compared to $6 \mathrm{~cm}$ group, as shown in Table (19).

Table (19): Predictors for minor complications

\begin{tabular}{|c|c|c|c|c|}
\hline & $\begin{array}{l}\text { p- } \\
\text { value } \\
\text { Lowe } \\
\mathbf{r}\end{array}$ & $\begin{array}{l}\text { Odds } \\
\text { ratio } \\
\text { Uppe } \\
\text { r }\end{array}$ & $\begin{array}{l}95.00 \\
\text { odds r } \\
\text { Lowe } \\
\text { r }\end{array}$ & $\begin{array}{l}\text { C.I. for } \\
\text { io } \\
\text { Upper }\end{array}$ \\
\hline $\begin{array}{l}\text { Groups } \\
(3 \mathrm{~cm} / 6 \mathrm{~cm} \\
)\end{array}$ & 0.014 & $\begin{array}{l}14.68 \\
4\end{array}$ & 1.722 & $\begin{array}{l}125.23 \\
9\end{array}$ \\
\hline Constant & 0.065 & 2.111 & & \\
\hline
\end{tabular}

\section{DISCUSSION}

LSG has become a very popular bariatric procedure because of the several advantages that it carries over other more complex procedures such as the laparoscopic LRYGB ${ }^{[8]}$ and, is gaining momentum as a definitive single-stage procedure for morbid obesity ${ }^{[4,9]}$.

The fundus is the most easily expanded compartment of the reservoir part since it has only two layers of muscle; enabling that way the stomach to accommodate larger volumes. Therefore, resecting the fundus during LSG results not only in volume capacity reduction, but also in removal of the most distensible part of the stomach leading to high intraluminal pressure and consequently to early satiety feeling ${ }^{[10]}$. All restrictive procedures show a tendency to weight gain after several years. This can be caused by adaptation to soft and liquid high-calorie food ingestion and/or loss of restriction ${ }^{[11]}$.

LSG may be followed by insufficient weight loss and or weight regain with or without recurrence of co-morbidities. The potential explanation for LSG failure may be eventually identified in the dilation of the gastric tube with consequent increase in the gastric capacity, an incomplete removal of the gastric fundus ${ }^{[8]}$.

One considers what the difference in the bougie size actually means. Taking under consideration that $1 \mathrm{Fr}$ equals $0.3 \mathrm{~mm}$, a bougie of $36 \mathrm{Fr}$ has $1.2 \mathrm{~cm}$ diameter and contains 26 $\mathrm{cm} 3$ volume, does not vary significantly from the $40 \mathrm{Fr}$ bougie $(1.3 \mathrm{~cm}$ diameter and $32 \mathrm{~cm} 3$ volume) ${ }^{[10]}$.

The size of the bougie used for calibrating the stomach tube might influence success, i.e., weight loss, but this correlation appears to be complex and is definitely not linear ${ }^{[12]}$.

In this study, we used a fixed bougie size $36 \mathrm{Fr}$ while changing the size of antral pouch $6 \mathrm{~cm}$ versus $3 \mathrm{~cm}$. We have proved that the $3 \mathrm{~cm}$ antral pouch group have higher rate of vomiting compared to $6 \mathrm{~cm}$ group with fixed bougie size $36 \mathrm{Fr}$ and that $3 \mathrm{~cm}$ groups were $>6$ times at a higher risk to have vomiting > once compared to $6 \mathrm{~cm}$ group. These results differed to some extent with Jacobs and his co-workers who reported that no statistically significant difference between 4 and $7 \mathrm{~cm}$ antral pouch existed and agreed with that no difference between 46-Fr, 40-Fr, and 36-Fr bougie regarding excess body weight loss EBWL. However, our results agreed with Jacobs and his co-workers regarding excess body weight loss that there was no differences in the results of $6 \mathrm{~cm}$ groups and $3 \mathrm{~cm}$ antral pouch ${ }^{[10,13]}$.

Most patients in this study were young adults and middle age; $21-40$ (63.3\%), females $(73.3 \%)$, married $(60 \%)$. This gives indication of the age, sex and marital status looking for this operation, most probably for functional and psychological elements. The majority of subjects in our study (53.4\%) were working and did the operation for improving their performance. Fifty percent of patients in this study had BMI $>50,26.7 \%$ had BMI 40-45\%, and $3.3 \%$ had BMI $35-40 \%$ with co-morbidities denoting that the majority of patients did not look for this operation until they became morbidly obese.

Ninety percent of the patients in this study were not diabetic versus $10 \%$ only who were diabetic maintained on oral hypoglycemic drugs with minimal improvement over the period of six months in the form of reduction of the doses of their oral hypoglycemic drugs. $83.3 \%$ of subjects were not hypertensive versus $16.7 \%$ were hypertensive and on medication. They showed minimal improvement over the period of six months in the form of reduction of the doses of anti-hypertensive drugs.

Excess BMI loss reached $62 \%$ at 1 year, while in this study, the overall patients' weight loss percentage ranged from 30 to $86.9 \%$ excess body weight loss with a mean of $60 \%$ at 6 months. In group A, patients' weight loss percentage ranged from 31.2 to $86.9 \%$ excess 
body weight loss with a mean of $60.9 \%$, and in group B, patients' weight loss percentage ranged from 30 to $83.5 \%$ excess body weight loss with a mean of $61.1 \%$. In this study, (40\%) of patients lost (41-60\%) of their excess body weight within 6 months without significance to any group

However, these results were not expected as the size of the pouch had increased with the $6 \mathrm{~cm}$ group and so more weight loss had expected to be with group a $(3 \mathrm{~cm})$. So, more time of follow-up may be needed to prove that small pouch is more effective for weight loss.

In this study, two patients (13.4\%) in group A lost more than $80 \%$ of their excess body weight over a period of six months versus one patient (6.6\%) in group B lost $80 \%$ of his excess body weight and that $93.3 \%$ lost $40-80 \%$ over the period of 6 months. However, this result had no significance and may be of significance if more patients were done. In addition, this study showed that the percentage of weight loss was more with lower basic BMI so the less was the basic BMI the more is the percentage of weight loss at 6 months.

Complications were graded according to the Clavien classification system ${ }^{[14]}$ : grade I, a complication inducing any deviation from the normal postoperative course; grade II, complications requiring pharmacologic treatment; grade III, complications requiring operative, endoscopic, or radiologic intervention; grade IV, life-threatening complications requiring intermediate or intensive care unit management; and grade V, death of a patient ${ }^{[15]}$.

In this study, there was no major complications (e.g.; leakage, bleeding, pulmonary embolism or death). Applying this grading in this study, we find that all complications belonged to grade 1 and 2 in the form of nausea, vomiting and reflux symptoms, where all responded to medical treatment and one case only of severe vomiting that was re-admitted to the hospital for IV fluids infusion and discharged after two days. We found that $96.9 \%$ (17 patients) in $3 \mathrm{~cm}$ group developed minor complications mostly reflux and vomiting and $67.9 \%$ (13 patients) in $6 \mathrm{~cm}$ group denoting that minor complications as nausea vomiting and reflux were strongly significant [p-value was (0.003)] to $3 \mathrm{~cm}$ group and our results proved that $3 \mathrm{~cm}$ groups were
$>14$ times at a higher risk to have these complications compared to $6 \mathrm{~cm}$ group.

In this study $60 \%$ of patients developed no reflux symptoms while $40 \%$ developed vomiting more than once so vomiting is related to the size of antral pouch where the $3 \mathrm{~cm}$ antral pouch group have higher rate of vomiting compared to $6 \mathrm{~cm}$ group with fixed bougie size $36 \mathrm{Fr}$ and that $3 \mathrm{~cm}$ groups were $>6$ times at a higher risk to have vomiting more than once compared to $6 \mathrm{~cm}$ group. Even WernickeKorsakoff syndrome has been reported after sleeve gastrectomy (SG) due to prolonged vomiting. Most authors report prescribing PPIs for different periods of time to the SG patients ${ }^{[3]}$; in this study PPI were prescribed routinely for all patients for 3 months at least, where only one case was re-admitted for severe vomiting and dehydration where she received IV fluids for 2 days and discharged after that.

Evangelos and his colleges ${ }^{[16]}$ mentioned that reinforcement of the stapling line is a negative predictor for subsequent complications, while a high preoperative BMI, previous bariatric operation, and diabetes are positive predictors which contradict the results in this study which stated that DM, hypertension and other chronic diseases had no influence on development of any post-operative nausea, vomiting or reflux.

\section{Conclusion}

$3 \mathrm{~cm}$ groups (group A) were $>14$ times at a higher risk to have minor complications in the form of nausea; vomiting and reflux compared to $6 \mathrm{~cm}$ group (group B) without any difference between both groups regarding BMI changes over a period of six months so we recommend leaving an antral pouch of $6 \mathrm{~cm}$ size while performing this procedure for better outcome with minor complications.

\section{REFERENCES:}

1.Krawczykowski $\quad \mathrm{D}(\mathbf{2 0 0 8})$ : $\quad$ La sleeve gastrectomy. Acta Endoscopica, 38(1): S57-S62.

2.Baltasar A, Serra $\mathrm{C}$ and Pérez $\mathrm{N}$ et al. (2005):Laparoscopic sleeve gastrectomy: a multi-purpose bariatric operation. Obesity surgery, 15(8): 1124-1128.

3.Deitel $M$, Crosby $R$ and Gagner $\mathbf{G}$ et al. (2007): The first international consensus summit for sleeve 
gastrectomy (SG), New York City.Obesity surgery, 18(5): 487-496.

4.Almogy G , Crookes P, Anthone G et al. (2004): Longitudinal gastrectomy as a treatment for the high-risk super-obese patient. Obesity surgery,14(4): 492497.

5.Regan J (2003): Early experience with twostage laparoscopic Roux-en-Y gastric bypass as an alternative in the supersuper obese patient. Obesity surgery, 13(6): 861-864.

6.Tucker O, Szomstein S, Rosenthal R et al. (2012): Indications for sleeve gastrectomy as a primary procedure for weight loss in the morbidly obese. Journal of Gastrointestinal Surgery,12(4): 635-675.

7. Melissas J, Koukouraki S and Askoxylakis J et al. (2008): Sleeve gastrectomy-a "food limiting" operation. Obesity surgery, 18(10): 1251-1256.

8. Antonio I, Anne $\mathrm{S}$, Patrick $\mathrm{N}$ et al. (2011):Re-sleeve gastrectomy for failed laparoscopic sleeve gastrectomy: a feasibility study. Obesity surgery, 21(7): 832-835.

9. Lalor PF, Tucker ON ,Szomstein S et al. (2008): Complications after laparoscopic sleeve gastrectomy. Surgery for Obesity and Related Diseases, 4(1): 33-38.

10.Joanna P, Konstantinos A ,Konstantinos G et al. ( 2010): Morbid obesity and sleeve gastrectomy: how does it work? Obesity surgery, 20(10): 1448-1455.

11. Rudolf A, Sylvia W, Ingmar $P$ et al. (2007): Laparoscopic sleeve gastrectomy-influence of sleeve size and resected gastric volume. Obesity surgery, 17(10): 1297.

12. Franz X, Felix L, Soheila S et al. ( 2008): Laparoscopic sleeve gastrectomy as an isolated bariatric procedure: intermediate-term results from a large series in three Austrian centers. Obesity surgery,18(7): 814-818.

13.Jacobs M, Bisland $W$ and Gomez $E$ et al. ( 2010): Laparoscopic sleeve gastrectomy: a retrospective review of 1-and 2-year results. Surgical endoscopy, 24(4): 781-785.

14.Dindo D, Demartines N, Clavien PA et al. (2004):Classification of surgical complications: a new proposal with evaluation in a cohort of 6336 patients and results of a survey. Annals of surgery, 240(2): 205.

15.Fuks D, Demartines $N$ and Clavien $P$ et al. (2009): Results of laparoscopic sleeve gastrectomy: a prospective study in 135 patients with morbid obesity. Surgery, 145(1): 106-113.

16.Evangelos M, Konstantinos M ,Konstantinos A et al. (2010): Laparoscopic sleeve gastrectomy performed with intent to treat morbid obesity: a prospective single-center study of 261 patients with a median follow-up of 1 year. Obesity surgery, 20(3): 276-282.

17.Masao K, Nobuo $Y$, Toshimasa $O$ et al. (2002) : Criteria and classification of obesity in Japan and Asia-Oceania Asia Pacific J Clin Nutr., 11: S732-S737 